\title{
Down regulation of cotton GbTRP1 leads to accumulation of anthranilates and confers resistance to Verticillium dahliae
}

\author{
MIAO Yuhuan ${ }^{1,2}$, ZHU Longfu $^{1}$ and ZHANG Xianlong ${ }^{1 *}$
}

\begin{abstract}
Background: Verticillium wilt, caused by Verticillium dahliae, is called a "cancer" disease of cotton. The discovery and identification of defense-related genes is essential for the breeding of Verticillium wilt-resistant varieties. In previous research we identified some possible broad-spectrum resistance genes. Here, we report a tryptophan synthesis-related gene GbTRP1 and its functional analysis in relation to the resistance of cotton to $V$. dahliae.

Results: Expression analysis shows that GbTRP1 is suppressed at $1 \mathrm{~h}$ and $6 \mathrm{~h}$ post $V$. dahliae infection, but activated at $12 \mathrm{~h}$ and $24 \mathrm{~h}$, and the expression of GbTRP1 is highly induced by treatment with salicylic acid and jasmonic acid. Sub-cellular localization studies show that GBTRP1 is localized in the chloroplast. Suppression of GbTRP1 expression leads to lesion-mimic phenotypes and activates the immune response in cotton by showing enhanced resistance to $V$. dahliae and $B$. cinerea. Metabolomic analysis shows that anthranilic compounds significantly accumulated in GSTRP1-silenced plants, and these metabolites can inhibit the growth of $V$. dahliae and B. cinerea in vitro,
\end{abstract}

Conclusions: Our results show that suppression of GbTRP1 expression dramatically activates the immune response and increases resistance of cotton to $\mathrm{V}$. dahliae and $B$. cinerea, possibly due to the accumulation of anthranilate compounds. This study not only provides genetic resources for disease resistance breeding, but also may provide a basis for new chemical control methods for combatting of fungal disease in cotton.

Keywords: Cotton, GBTRP1, Verticillium dahliae, Botrytis cinerea, Anthranilate compounds

\section{Background}

Cotton is an important natural fiber crop in the textile industry and the economic pillar of many developing countries. Verticillium wilt is a disease caused by Verticillium dahliae and is becoming a worldwide threat to the production of cotton. The disease was first discovered in Virginia in 1914 (Carpenter 1914), and spread to China along with the introduction of Stoneville cotton in 1935 (Cai et al. 2009). The typical symptoms of diseased cotton are yellowing, wilting and leaf drop, which greatly reduces the yield and quality of cotton fiber. However, there are almost no disease-resistant germplasm resources in upland cotton, which accounts for the $95 \%$ of the total cotton yield in China.

\footnotetext{
*Correspondence: xlzhang@mail.hzau.edu.cn

${ }^{1}$ National Key Laboratory of Crop Genetic Improvement, Huazhong

Agricultural University, Wuhan 430070, Hubei, China

Full list of author information is available at the end of the article
}

The resistance mechanism of most host plants to $V$. dahliae is still obscure, with the exception of tomato. The Ve1 gene from tomato was successfully identified through map-based cloning and shown to confer specific resistance to the race 1 strain of $V$. dahliae (Kawchuk et al. 2001). Although tomato Ve1 conferred resistance to V. dahliae race 1 in Arabidopsis (Fradin et al. 2011), it failed to improve cotton resistance to $V$. dahliae when ectopically expressed in cotton, due to the absence of the avirulence gene ave1 in $V$. dahliae from cotton (Liu et al. 2014a; Song et al. 2018). In the last few years, several signalling pathways have been identified to play a role in cotton defense response by using transcriptomic and proteomic approaches, including homeostasis of reactive oxygen species and hormone signalling pathways such as salicylic acid, jasmonic acid and ethylene signal pathways (Xu et al. 2011; Gao et al. 2013; Xu et al. 2014; $\mathrm{Li}$ et al. 2016). GbWRKY1(Li et al. 2014), GhSSN (Sun 
et al. 2014), GhJAZ2 (He et al. 2017), GhLAC1 (Hu et al. 2018a), GbERF1 (Guo et al. 2016), GhNDR1, GhMKK2 (Gao et al. 2011) amongst others have been identified as important genes involved in the regulation of hormone synthesis and defensive signal transduction pathways, and influence cotton resistance to $V$. dahliae. In addition, some natural products called phytoalexins, such as gossypol and flavonoids (Mace et al. 1985; Hu et al. 2018a), are chemical weapons in cotton that play an important role in inhibiting the growth and reproduction of $V$. dahliae. However, these secondary metabolites and their synthetic pathways have not been studied in detail in cotton.

Tryptophan is not only an amino acid for protein synthesis, but also an important precursor of auxin and other secondary metabolites. These metabolites play an essential role in plant growth and defense response (Chen et al. 2007; Bednarek et al. 2009; Iven et al. 2012). In a previous study, we found that downregulation of tryptophan synthase GbTSA1 could increase cotton resistance to $V$. dahliae. The indolic metabolites derived from the tryptophan pathway have the capacity to elicit defense responses and enhance cotton resistance to $V$. dahliae (Miao et al. 2019).

In this study, we found that suppression of GbTRP1 expression, encoding an phosphoribosylanthranilate transferase (PAT, EC2. 4. 2. 18) in tryptophan synthesis pathway, dramatically activated the expression of defense-related genes and led to a spontaneous cell death phenotype in cotton. Metabolomics analysis showed that anthranilate compounds including $\mathrm{N}$-benzylformamide, methyl anthranilate, 1-hydroxy-2-indolinone and $\mathrm{N}$-acetylanthranilic acid accumulated to high levels in GbTRP1-knockdown plants. These compounds have antifungal or cytotoxic activities and play a role in the resistance of cotton to $V$. dahliae and $B$. cinerea. This study not only provides genetic resources for disease resistance breeding, but also may provide a basis for new chemical control methods for combatting fungal disease in cotton.

\section{Results}

Expression pattern analysis of GbTRP1

GbTRP1 was identified previously from a data-mining strategy and showed response to a broad range of pathogens (Xu et al. 2014; Miao et al. 2019). The protein sequence of GbTRP1 is 410 amino acids in length and contains a chloroplast transit peptide (cTP) and an anthranilate phosphoribosyl transferase domain (Fig. 1a), which shares a high similarity with AtTRP1 (Additional file 1: Figure S1). Therefore, GbTRP1 was recognized as a phosphoribosyl anthranilate transferase and catalyzed the second step of tryptophan synthesis. Following inoculation of cotton plants with $V$. dahliae strain "V991", the transcript levels of GBTRP1 decreased at $1 \mathrm{~h}$ and $6 \mathrm{~h}$ but increased at $12 \mathrm{~h}$ and $24 \mathrm{~h}$ post infection. Salicylic acid (SA) and jasmonic acid (JA) are two defensive hormones involved in resistance of cotton to V. dahliae (Liu et al. 2014b; He et al. 2017; Hu

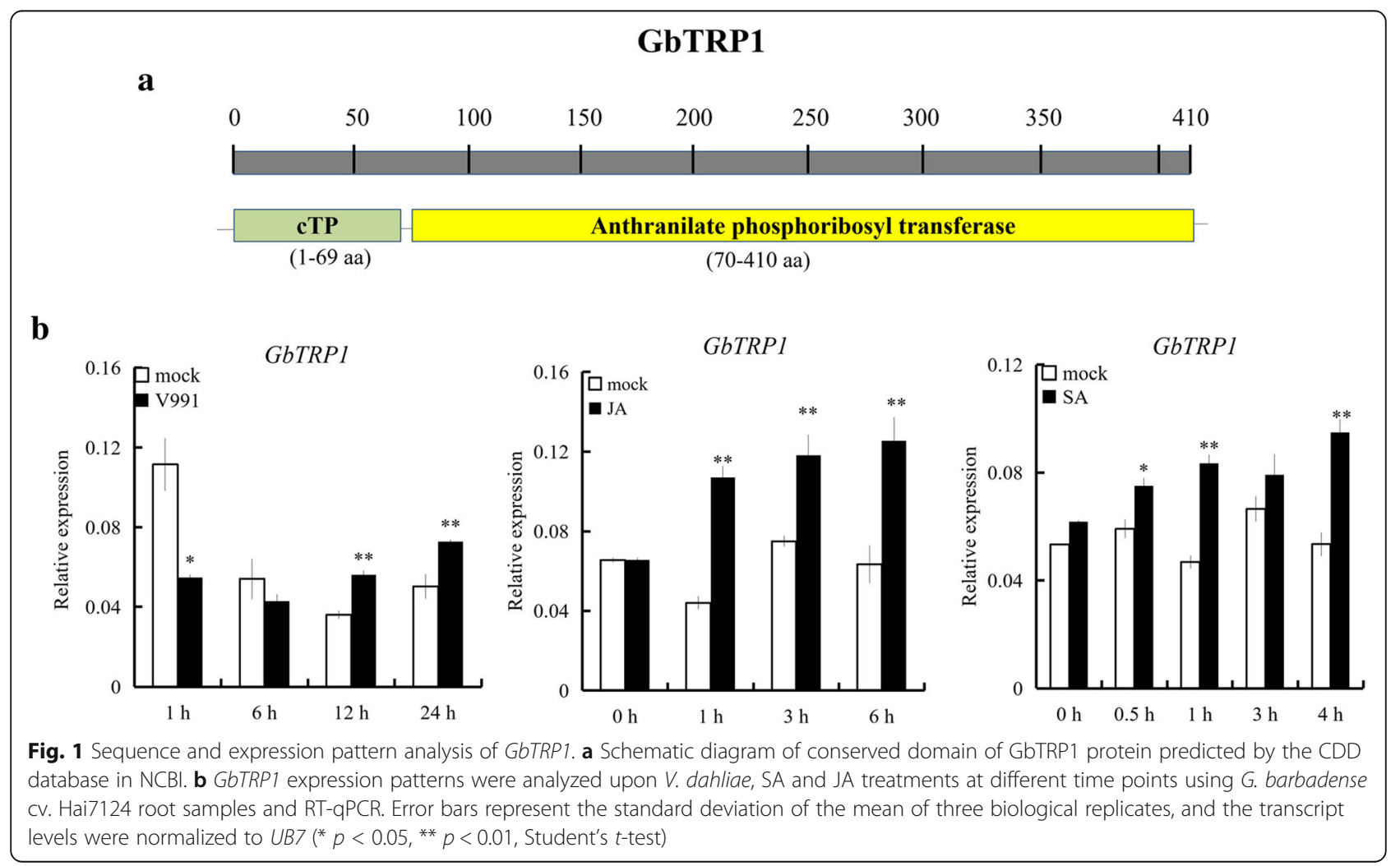


et al. 2018b; Miao et al. 2019). We found that GbTRP1 was highly induced by SA and JA treatment (Fig. 1b). These results suggest that GbTRP1 might play a role in the defense response of cotton to $V$. dahliae. GbTRP1 protein contains cTP in the N-terminus of the protein, which indicates that GbTRP1 might be localized in the chloroplast. To confirm this, we constructed the GbTRP1-GFP fusion protein driven by CaMV35S promoter and transiently expressed the vector in the tobacco leaf cells to observe the GFP fluorescence by confocal laser scanning microscope. The fluorescence of GbTRP1-GFP fusion protein colocalized with the spontaneous fluorescence of chloroplast, while the control vector containing GFP protein with no CTP mainly localized to the nucleus and membrane (Fig. 2). These results showed that GbTRP1 protein was localized in the chloroplast as predicted.

\section{Suppression of GbTRP1 expression triggers cell death and defense response in cotton}

To validate the function of GbTRP1 in cotton resistance to $V$. dahliae, we obtained three transgenic lines ( $p i-1$, $p i-3, p i-4)$ in which GbTRP1 expression was suppressed by RNAi. Cell death and lesion mimic phenotypes were observed on the stems of GbTRP1-RNAi transgenic plants under normal (uninfected) growth conditions (Fig. 3a). The young leaves of wild type (WT) and transgenic plants were sampled to detect the expression levels of GbTRP1 and the results showed that the mRNA amount of GbTRP1 was greatly decreased in transgenic plants compared with WT control plants (Fig. 3b). It has been reported that many lesion mimic mutants are coupled with a high expression level of $P R$ genes (Sun et al. 2014; Chai et al. 2017). To investigate this in GbTRP1-RNAi plants, we analysed the transcript levels for PR1, PR2 and PR5 and found that the expression levels of $P R$ genes were significantly increased in the RNAi plants (Fig. 3c). We also knocked-down the expression of GbTRP1 using the VIGS technology and the phenotypes of the TRV:TRP1 plants were similar to the RNAi plants. The TRV:TRP1 plants were stunted, with lesion mimics occurring on the stems and leaves (Fig. 4a). RT-PCR was used to analyse the expression levels of GbTRP1 in TRV:00 and TRV:TRP1 plants, and the results showed that the expression of GbTRP1 was greatly suppressed in TRV:TRP1 compared with TRV:00 plants (Fig. 4b). The expression of $P R$ genes was monitored in the young leaves 2 weeks post VIGS infiltration before the appearance of lesions. All the $P R$ genes examined were strongly up-regulated in TRV:TRP1 plants compared with control plants (Fig. 4c). Therefore, suppressing the expression of GbTRP1 activates the immune response in cotton in the absence of pathogens.

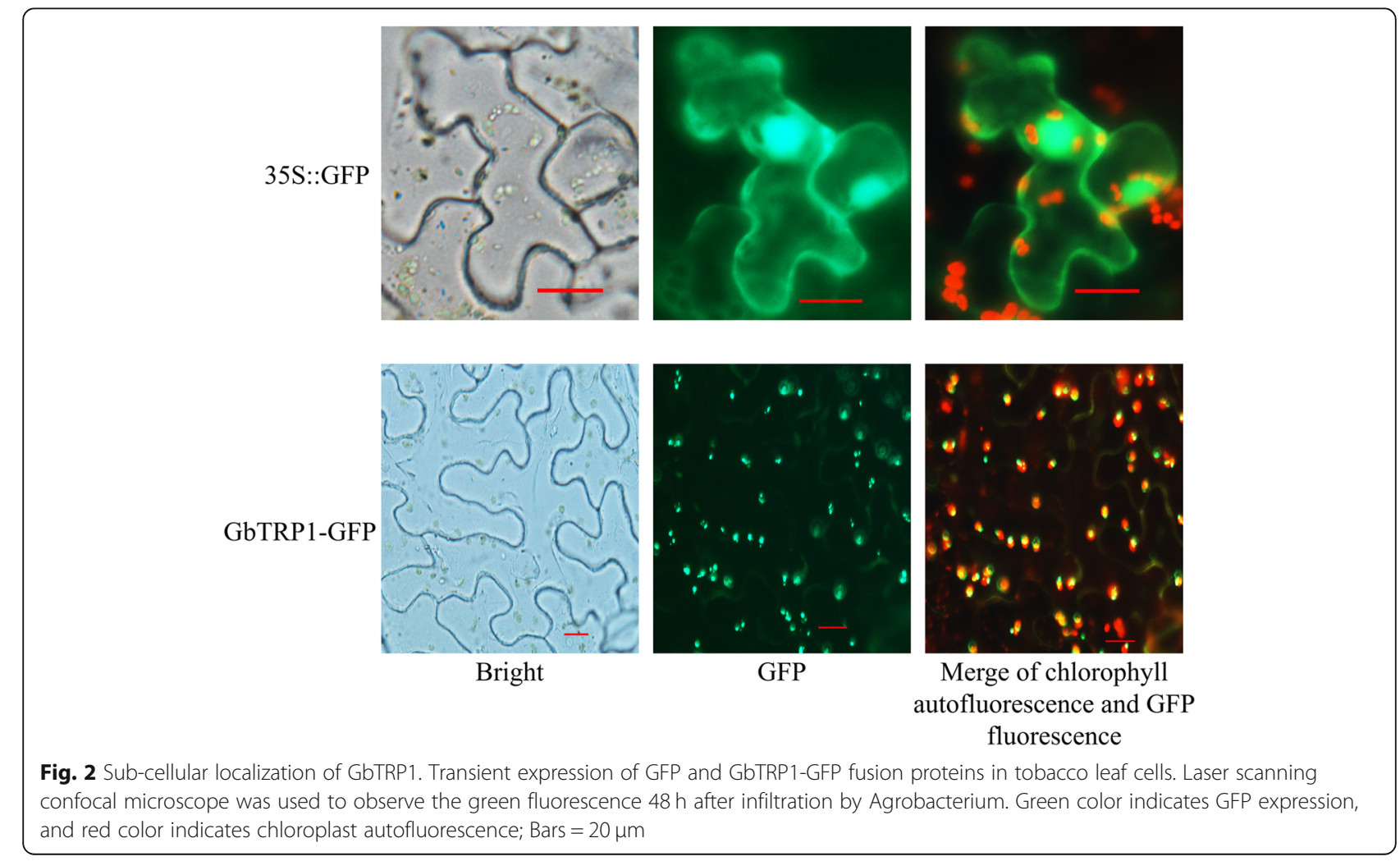


$\mathbf{a}$
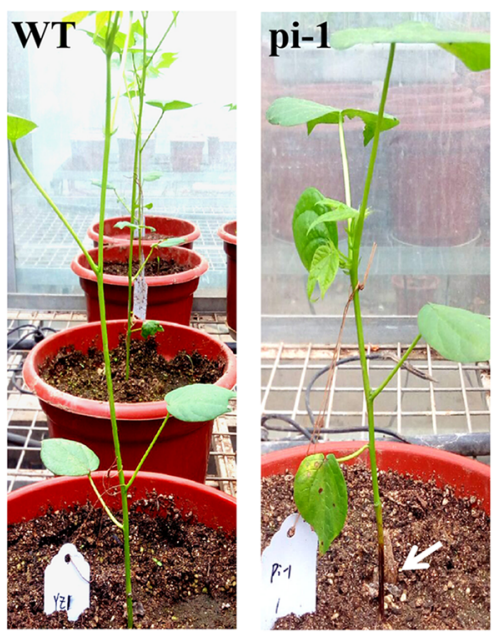

c

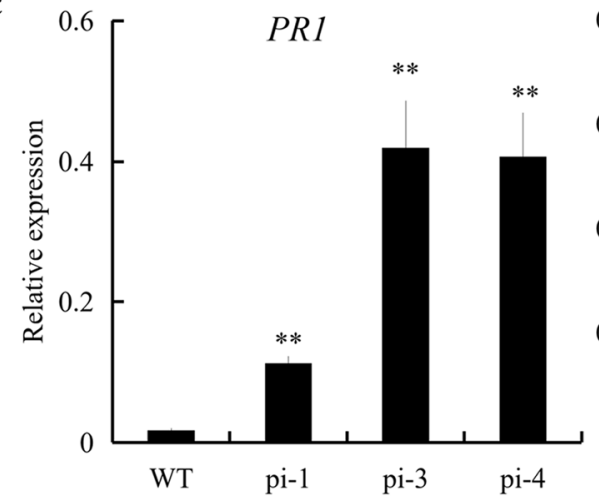

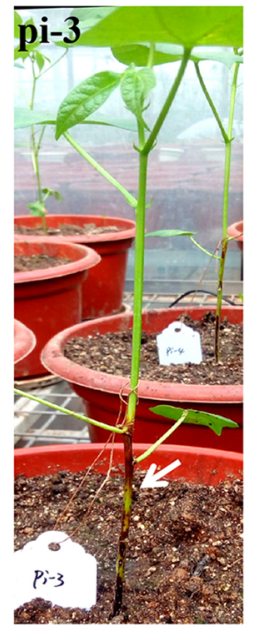
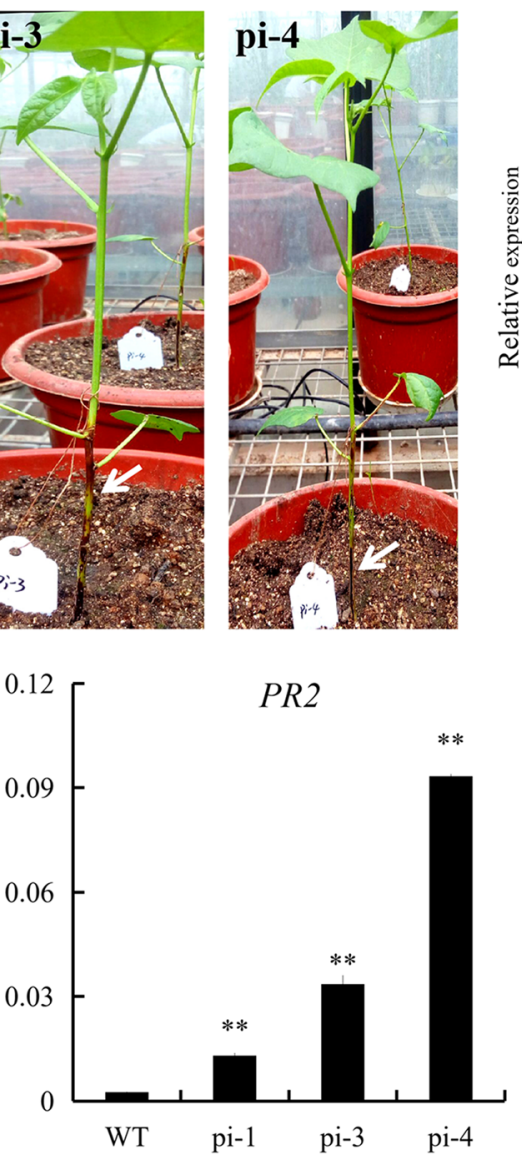

b
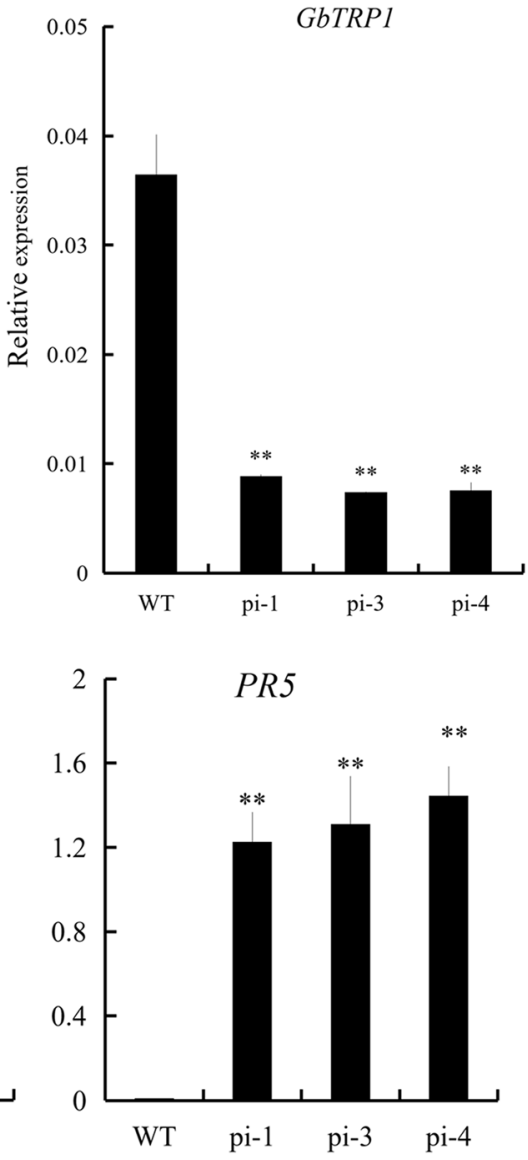

Fig. 3 Phenotypic observation and expression analysis of GbTRP1-RNAi plants. a Lesion-mimic phenotypes were observed on the stems of GbTRP1-RNAi transgenic lines. Plants were grown in the greenhouse for 2 months and the white arrows indicate the sites of necrosis. $\mathbf{b}$ and $\mathbf{c}$ RTQPCR analysis of the expression levels of GbTRP1 and PR genes in WT and GbTRP1-RNAi lines in 10 day-old plants before the lesions appeared on the plants. Error bars represent the standard deviation of the mean of three biological replicates (** $p<0.01$, Student's $t$-test)

Suppression of GbTRP1 expression enhances resistance of cotton to $V$. dahliae and $B$. cinerea

To examine pathogen resistance of GbTRP1-knockdown plants, we selected the plants with relatively mild symptoms of necrosis and inoculated these with $V$. dahliae or $B$. cinerea. The results show that resistance of the transgenic plants (pi-1, pi-3, pi-4) to $V$. dahliae was enhanced, with the plants showing reduced leaf chlorosis, fewer necrotic vascular bundles and a lower disease index compared with control plants (Fig. 5a, b, c).

TRP1 was also responsive to $B$. cinerea infection in Arabidopsis (Xu et al. 2014). To investigate the role of GbTRP1 in cotton resistance to $B$. cinerea, the leaves of TRV:00 and TRV:TRP1 plants were collected at 16 days post VIGS infiltration and inoculated with $B$. cinerea, and disease symptoms were observed 7 days post inoculation. The detached leaves from TRV:00 plants exhibited severe necrosis while leaves from TRV:TRP1 showed less necrosis; the disease symptom area measured by Image J software also supported this observation (Fig. 6a, b). These results show that suppressing the expression of GbTRP1 enhances the resistance of cotton to both $V$. dahliae and B. cinerea.

\section{Anthranilate compounds accumulate to high levels in TRV:TRP1 plants}

We found that the transgenic RNAi plants (pi-1, pi-3, pi-4) and TRV:TRP1 plants have an aromatic smell under normal conditions and exhibit blue fluorescence under UV light (Fig. 7a). These phenotypes are similar to the fluorescent and aromatic trp1 mutants which accumulate a large amount of anthranilic acid and anthranilate $\beta$-glucoside (Last and Fink 1988). Gas chromatography-mass spectrometry (GC-MS) were used to investigate the possible changes in metabolite accumulation in TRV:00 and TRV:TRP1 seedlings. The results show that anthranilic acid and its 

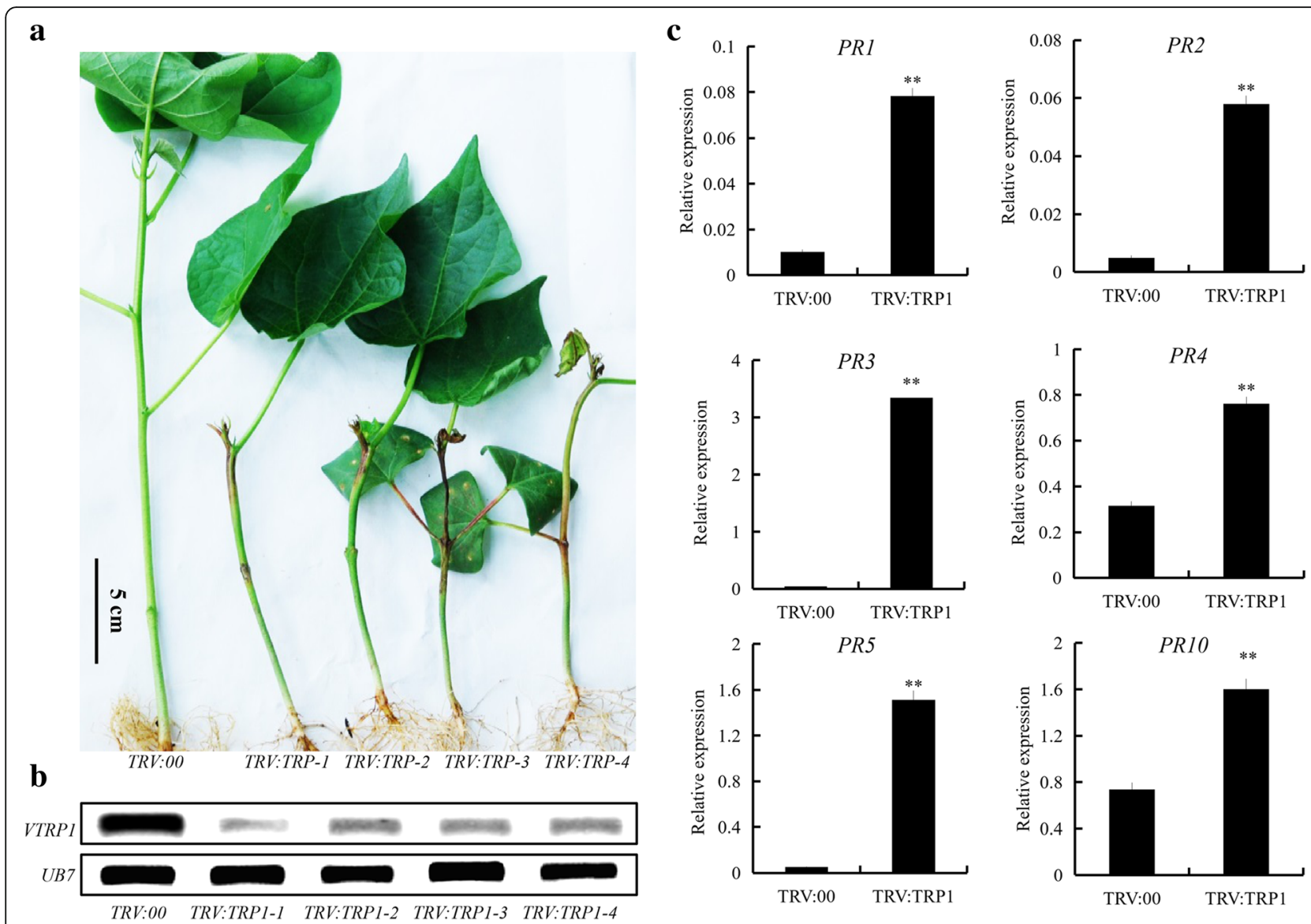

Fig. 4 Phenotypic observation and expression analysis of TRV:TRP1 plants. a Spontaneous lesion formation on the stems and young leaves of TRV:TRP1 cotton seedlings. Images were obtained 21 days after VIGS; Bars $=5 \mathrm{~cm}$. b RT-PCR analysis indicates that the transcripts of GbTRP1 are reduced at 14 days after infiltration (** $p<0.01$, Student's $t$-test). c RT-qPCR to detect the transcript levels of PR genes in TRV:TRP1 and control plants 14 days after infiltration, before the appearance of lesions. Error bars represent the standard deviation of the mean of three biological replicates $\left({ }^{* *} p<0.01\right.$, Student's $t$-test)

derivatives, including N-benzylformamide, methyl anthranilate, 1-hydroxy-2-indolinone and $\mathrm{N}$-acetylanthranilic acid, were accumulated to high levels in TRV:TRP1 transgenics, while these compounds were not detected in the control plants (Fig. 7b, c).

\section{Anthranilate compounds inhibit the growth of $V$. dahliae and $B$. cinerea}

To explore the possible biological function of elevated anthranilate compounds in TRV:TRP1 plants, we tested the toxicity of methyl anthranilate both to $V$. dahliae and B. cinerea. $V$. dahliae and B. cinerea were inoculated onto the potato dextrose agar (PDA) medium containing different concentrations $\left(0,100 \mu \mathrm{mol} \cdot \mathrm{L}^{-1}, 500 \mu \mathrm{mol} \cdot \mathrm{L}^{-1}\right.$, $1 \mathrm{mmol} \cdot \mathrm{L}^{-1}$ ) of methyl anthranilate, respectively. It was found that methyl anthranilate application greatly reduced the growth of $B$. cinerea at $100 \mu \mathrm{mol} \cdot \mathrm{L}^{-1}$ concentration and totally blocked the growth of $B$. cinerea at $500 \mu \mathrm{mol} \cdot \mathrm{L}^{-1}$ and $10 \mathrm{mmol} \cdot \mathrm{L}^{-1}$ (Fig. 8a, b). Six isolates of $V$. dahliae were selected to test the antifungal properties of methyl anthranilate. These strains can be divided into two groups according to their pathogenicity: the first group, comprising T9, 4TM6-15 and V991, are strong pathogenic isolates; the second, $1 \mathrm{~cd} 3-2, \mathrm{BP} 2$, and $1 \mathrm{HN}-1$, are weak pathogenic isolates. The results show that methyl anthranilate is toxic to all the six isolates of $V$. dahliae at $1 \mathrm{mmol} \cdot \mathrm{L}^{-1}$ concentration. The $V$. dahliae pathogens with low pathogenicity $(1 \mathrm{~cd} 3-2, \mathrm{BP} 2$, and $1 \mathrm{HN}-1$ ) were more susceptible to methyl anthranilate treatment than the strains with strong pathogenicity (T9, 4TM6-15 and V991) (Fig. 8c, d). These results show that anthranilate compounds may contribute to cotton resistance to $V$. dahliae and $B$. cinerea.

\section{Discussion}

Reduced expression of GbTRP1 leads to lesion-mimic phenotypes and activates the immune response in cotton Research on the tryptophan synthesis pathway was first carried out in E. coli, from which the tryptophan operon and attenuator models were proposed (Crawford 1989), 


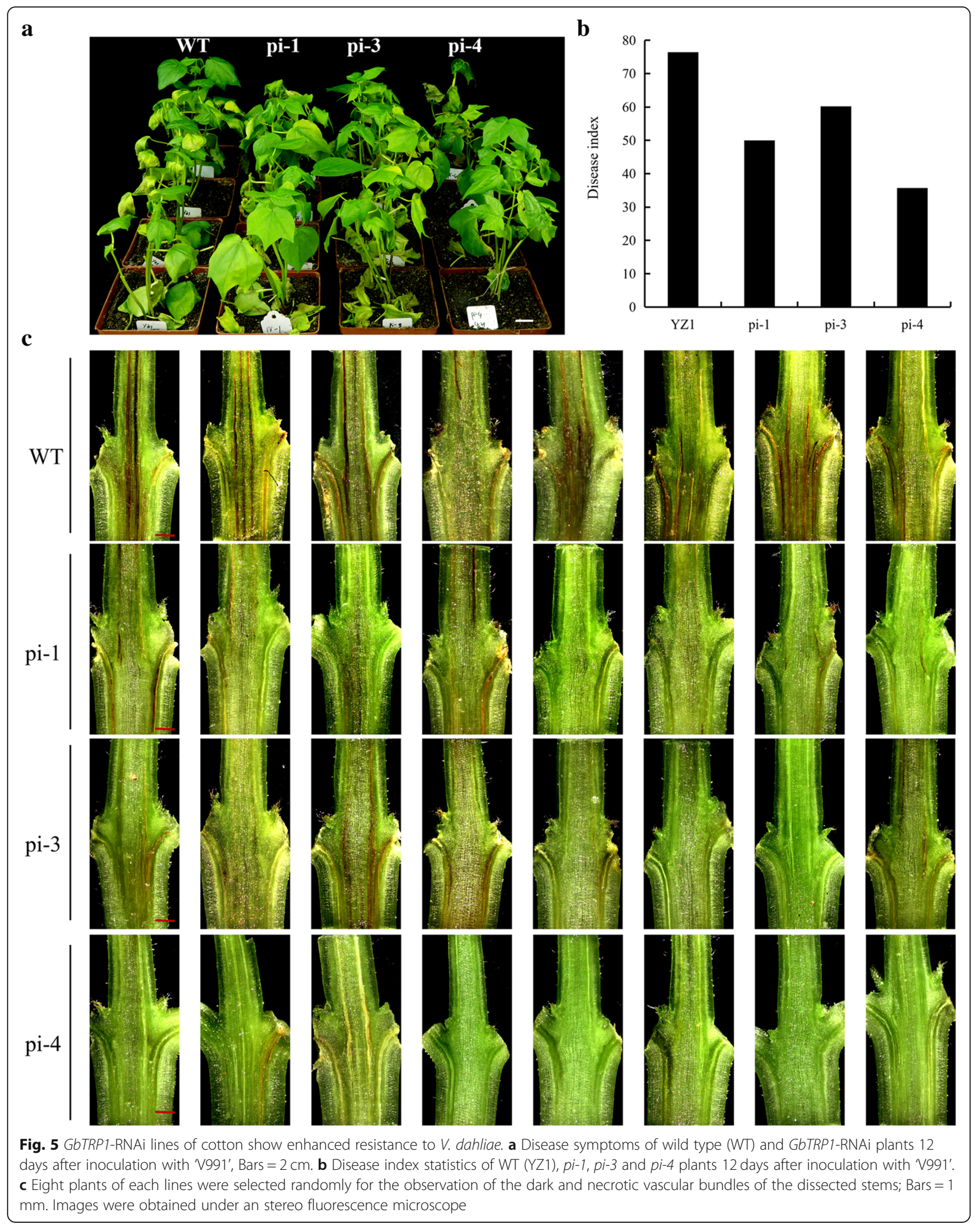




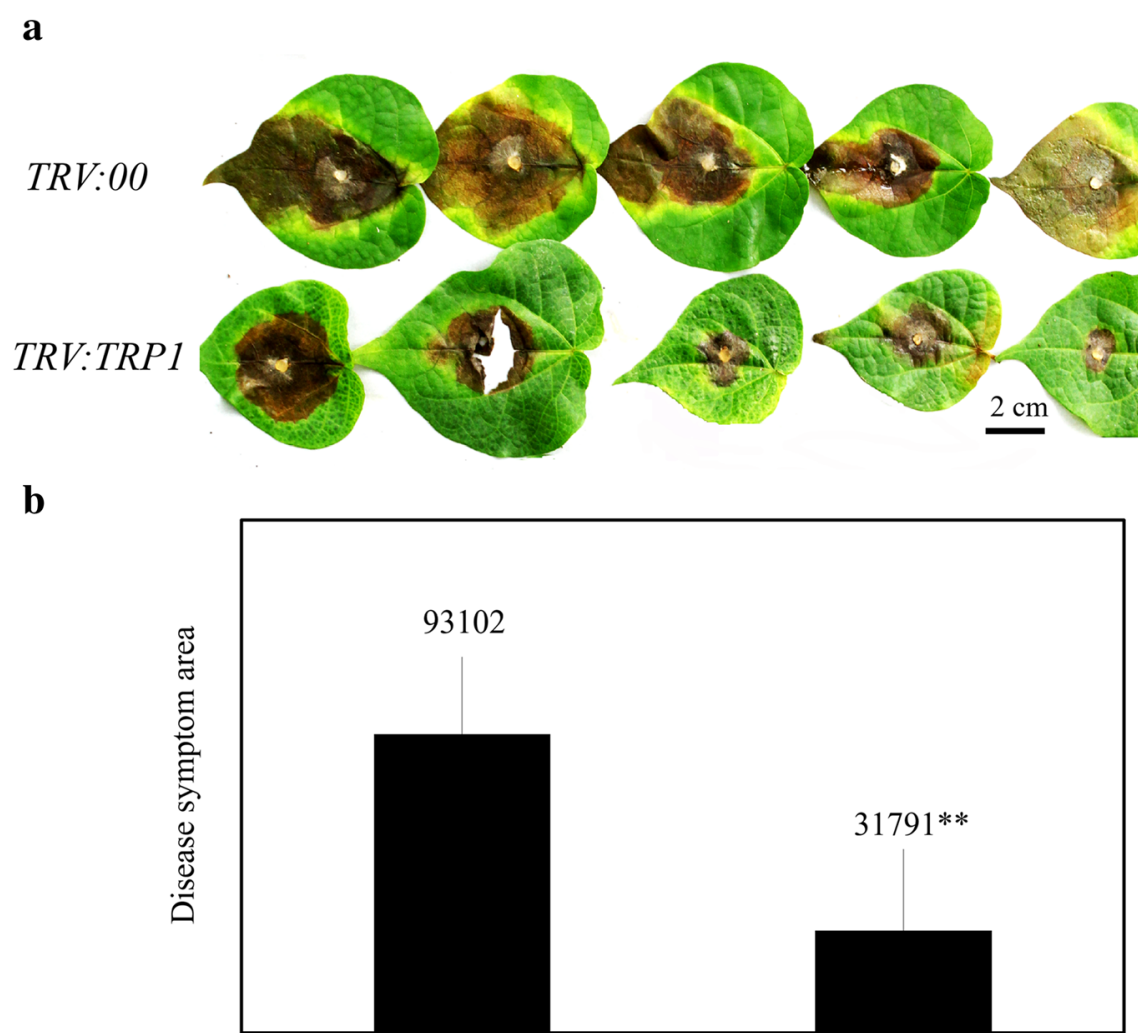

Fig. 6 TRV:TRP1 plants of cotton show enhanced resistance to B. cinerea. a Disease symptoms of TRV:00 and TRV:TRP1 leaves 7 days post inoculation with $B$. cinerea, Bars $=2 \mathrm{~cm}$. $\mathbf{b}$ The statistics of disease area by Image J software. The values represent the means from three biological replicates $(* * P<0.01$, Student's $t$-test)

and have become classic models of gene regulation in the history of molecular biology. Although the importance of tryptophan and its metabolites has been well understood for a long time in E. coli and in fungi, detailed studies of the tryptophan synthesis pathway in plants were not conducted until the 1990s. In Arabidopsis there are seven genes involved in Trp synthesis and all seven genes have been cloned (Radwanski and Last 1995). AtTRP1 was the first gene studied in tryptophan synthesis through mutant selection. The trp1 mutant of Arabidopsis is defective in phosphoribosyl anthranilate transferase (PAT) activity, and exhibits blue fluorescence under UV light because of the accumulation of anthranilate compounds. The trp1 mutant is also small and bushy, consistent with a defect in IAA biosynthesis (Last and Fink 1988; Rose et al. 1992).

We generated knock-down mutants of GbTRP1 through RNAi and VIGS technologies in cotton. Consistent with the Arabidopsis mutant phenotype, the GbTRP1-knockdown plants were also small and displayed blue fluorescence under UV light (Figs. 4a, and 7a). Interestingly, there were some different phenotypes observed in cotton from those of the tryptophan auxotroph mutants in Arabidopsis. GbTRP1-silenced plants exhibited spontaneous lesion mimics on the stems without pathogen infection (Figs. 3a and 4a), which was not reported in Arabidopsis trp1 mutants. We also found that several $P R$ genes including, $P R 1$, $P R 2$, and $P R 5$, were significantly activated in GbTRP1 RNAi and VIGS plants (Figs. 3c and 4c). These PR genes are usually associated with the activation of SA synthesis and signalling pathways (Van Loon and Van Strien 1999). However, the content of SA in TRV:TRP1 was less than that in TRV:00 plants in this study (Additional file 1: Figure S2) and in our previous study (Miao et al. 2019). There were three extra peaks in the SA chromatograms in TRV: TRP1 leaf samples, indicating that there may be new derivatives of SA accumulated in these mutants (Additional file 1: Figure S2). The mechanism for lesion-mimic phenotypes and activated immune response needs further study. We also found that GbTRP1 RNAi and TRV:TRP1 plants showed enhanced disease resistance to both $V$. dahliae and B. cinerea (Figs. 5 and 6), which suggested that down regulation of GBTRP1 may confer broad-spectrum resistance in cotton.

\section{Anthranilates have antifungal properties}

Plants produce large amounts of secondary metabolites and many of them are chemical weapons for plants to 


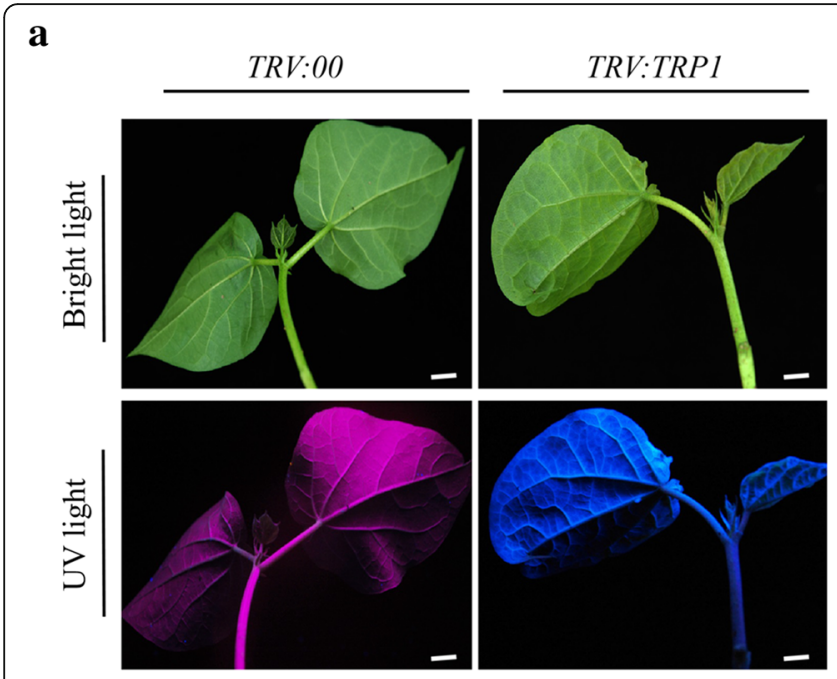

b

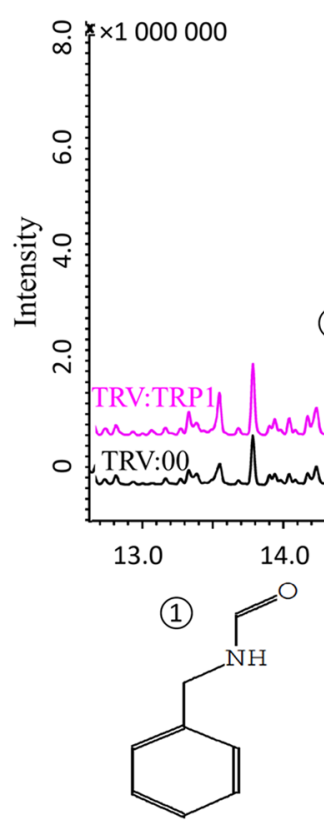

1:N-Benzylformamide
(1)

(2) c

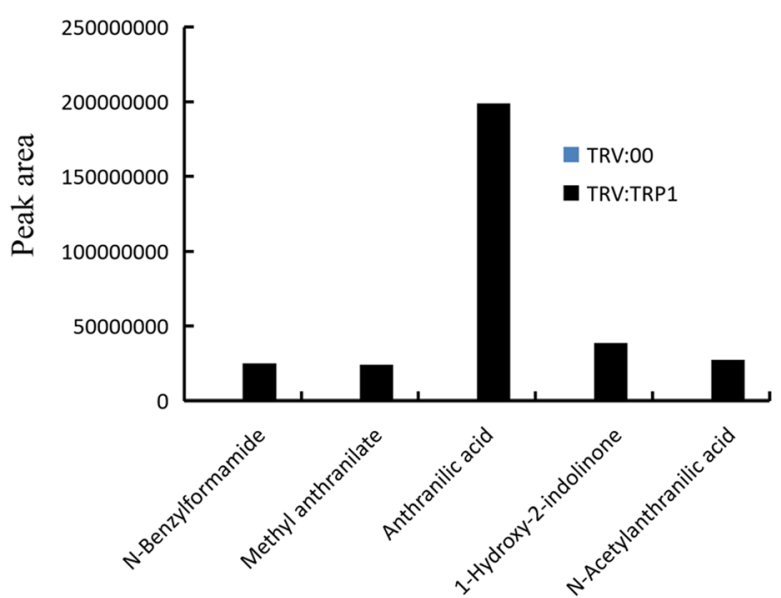

Fig. 7 Anthranilate metabolites accumulation in TRV:TRP1 seedlings detected by GC-MS. a Blue fluorescence phenotypes of TRV:TRP1 plants under UV radiation. Images were obtained 14 days after VIGS; Bars $=1 \mathrm{~cm}$. b Total ions chromatograph (TIC) and mass spectrum for measurement of metabolites by GC-MS in TRV:00 and TRV:TRP1 plants, and five extra peaks (retention time, RT =14.38, 14.95, 15.8, 19.7, 20.05) are indicated in TRV:TRP1 samples. c Quantification of anthranilic acid and its derivatives in TRV:00 and TRV:TRP1 plants

defend against the adverse environmental stresses. These secondary metabolites are usually derived from primary metabolites, including amino acids (Zeier 2013). Some compounds derived from amino acid metabolic pathways can act as defense signals, such as the well known methionine-derived hormone ethylene and the recently identified lysine catabolites pipecolic acid (Pip) and Nhydroxypipecolic acid (NHP) (Chae and Kieber 2005; Hartmann et al. 2018; Shan and He 2018). Other products derived from amino acid metabolic pathways, the phytoalexins, have antimicrobial and toxic activities that can directly protect plants from pathogen and insect attack (Ahuja et al. 2012).

Tryptophan is an essential amino acid that is a precursor of the hormone auxin and a large array of phytoalexins, such as glucosinolates, alkaloids and camalexin (Bednarek et al. 2009; Iven et al. 2012; Møldrup et al. 2013). Recently, Trp-derived serotonin has been reported that may facilitate insect performance on rice ( $\mathrm{Lu}$ et al. 2018). These compounds are all derived from the tryptophan metabolic 


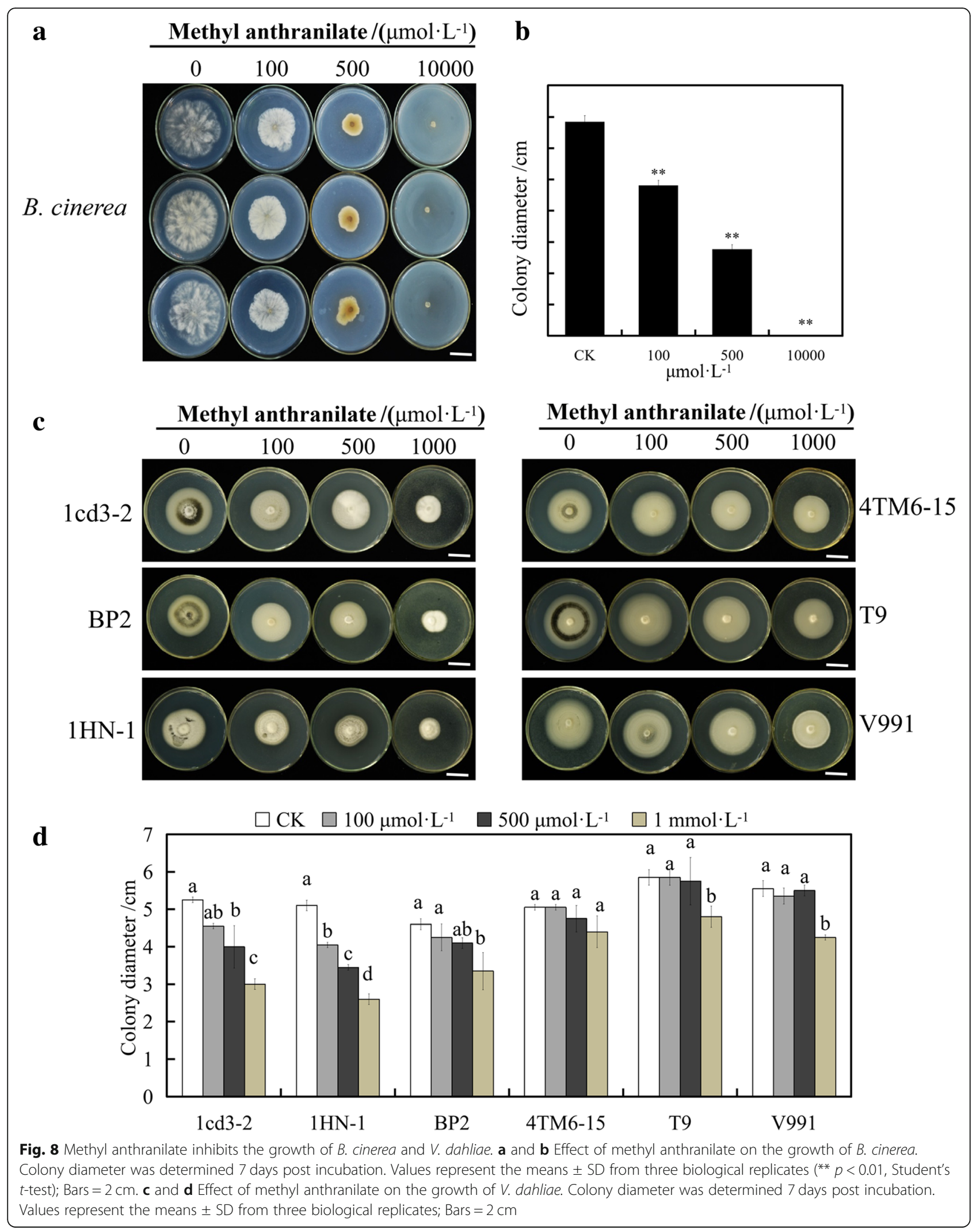


pathway, while, compounds from the tryptophan synthetic pathway have been less studied. Previously we found that knockdown of the expression of GbTSA1 and GbTSB1 led to the accumulation of indole and indolic metabolites, which can trigger the immune response during cotton resistance to $V$. dahliae (Miao et al. 2019). In this study, we show that suppression of GbTRP1 expression results in the accumulation of anthranilates with antifungal activity against $V$. dahliae and B. cinerea (Fig. 8). To our knowledge, the function of anthranilate compounds has not been studied in relation to plants resistance to pathogens. Further, these compounds may provide a basis for the chemical control of fungal disease in cotton. In addition to anthranilic acid, other anthranilic derivates including anthranilate $\beta$-glucoside, $\mathrm{N}$-benzylformamide, methyl anthranilate and $\mathrm{N}$-acetylanthranilic acid were also increased in GbTRP1 knockdown plants (Fig. 7). These results suggest that the activity of some enzymes such as glycosyltransferase, methyltransferase and acyltransferase may be activated in GbTRP1 knockdown plants and the genes encoding these enzymes require further study. These studies will help us to understand better the regulation of the aromatic secondary metabolic synthesis pathway, through the identification of key rate-limiting enzymes and new metabolic products may contribute to the engineering of pathogen-resistant crops.

\section{Conclusions}

Our results showed that suppression of GbTRP1 expression dramatically activated the immune response and increased resistance of cotton to $V$. dahliae and $B$. cinerea possibly due to the accumulation of anthranilate compounds. This study not only provides gene resources for disease resistance breeding, but also may provide a theoretical basis for chemical control of fungal disease in cotton.

\section{Materials and methods}

\section{Plant material and fungus proliferation}

Cotton plants, G. hirsutum cv. YZ1, G. barbadense cv. H7124 and transgenic plants derived from YZ1, were grown in the greenhouse under a controlled $14 \mathrm{~h}$ light/ $10 \mathrm{~h}$ dark cycle at $28^{\circ} \mathrm{C}$. For fungus proliferation, $V$. dahliae strain "V991" were first grown on potato dextrose agar (PDA) medium for 3 days, then the activated mycelia were cultured in Czapek's medium for 3-5 days at $25^{\circ} \mathrm{C}$ for spore production. B. cinerea were cultivated twice on PDA medium for activation.

\section{Stresses treatment and sample collection}

For $V$. dahliae treatment, seedlings of $\mathrm{H} 7124$ were cultivated in Hoagland solution for 2 weeks; the plants were root-dip-infected with $V$. dahliae conidial suspensions $\left(1 \times 10^{6}\right.$ conidia. $\left.\mathrm{mL}^{-1}\right)$ and the roots were harvested at
$1,6,12$, and $24 \mathrm{~h}$ after inoculation with control seedlings treated with sterile water. For hormone treatments, 1 $\mathrm{mmol} \cdot \mathrm{L}^{-1}$ SA and $100 \mu \mathrm{mol} \cdot \mathrm{L}^{-1}$ methyl jasmonate (MeJA) were applied in Hoagland solution independently, and root samples were collected at different time points after hormone treatment for analysis. At least 5 plants were sampled for each time point, with three biological repeats. All the samples were frozen in liquid nitrogen immediately after collection and stored at $80^{\circ} \mathrm{C}$ for subsequent RNA extraction.

\section{RT-qPCR analysis}

RNA was extracted according to the methods described previously (Zhu et al. 2005), and $3 \mu \mathrm{g}$ of total RNA was reverse-transcribed into cDNA for gene expression analysis. Reverse transcription-polymerase chain reaction (RT-PCR) and quantitative RT-PCR (qRT-PCR) were performed using the ABI Prism 7500 system (Applied Biosystems, Foster City, CA, USA) by the methods described by Miao et al. (2019).

\section{Vectors construction and genetic transformation}

To study the function of GbTRP1, a 464 bp fragment of GbTRP1 was amplified from the cDNA made from $G$. barbadense H7124 root samples and the PCR products were cloned into pHellsgate4 and pTRV2 to generate GbTRP1-RNAi and TRV:TRP1 vectors, respectively. For subcellular localization of GbTRP1 protein, the full-length cDNA sequence lacking stop codon was fused into the $\mathrm{N}$ terminus of green fluorescent protein (GFP) to construct 35S::GbTRP1-GFP. The primer sequences are listed in Additional file 1: Table S1. All vectors were transferred to Agrobacterium tumefacien (GV3101).

For genetic transformation, a GbTRP1-RNAi vector was used to transform hypocotyl sections of YZ1 using the Agrobacterium tumefaciens (GV3101)-mediated transformation methods described by Jin et al. (2006).

\section{Subcellular localization analysis}

A. tumefaciens harboring 35S::GbTRP1-GFP vector were transiently transformed into tobacco leaf cells using the methods described previously (Miao et al. 2019). The fluorescence of GFP was observed by confocal laser scanning microscopy (Olympus FV1200).

\section{Virus-induced gene silencing and pathogen inoculation}

A. tumefaciens harboring TRV:TRP1 and TRV:00 control vector were infiltrated into the cotyledons of 10 day-old H7124 seedlings using methods described previously (Gao et al. 2013). Leaf samples were then taken at 16 days post-VIGS infiltration for $B$. cinerea inoculation. The activated $B$. cinerea hyphal discs $(5 \mathrm{~mm})$ were inoculated onto the excised leaves at $25^{\circ} \mathrm{C}$ and then covered with black plastic wrap to maintain sufficient moisture. 
The lesion areas were measured by Image J software after 7 days post inoculation.

\section{Anthranilate assays}

Leaf samples were harvested from TRV:00 and TRV: TRP1 plants at 16 days post VIGS infiltration and immediately frozen in liquid nitrogen for determination of metabolites by gas chromatography-mass spectrometry (GC-MS). The methods for extraction and measurement of anthranilates are the same as the methods for indole determination (Miao et al. 2019).

\section{Determination of toxicity of methyl anthranilate to $V$. dahliae and $B$. cinerea}

To study the antifungal properties of anthranilates, methyl anthranilate was used as an exemplar. PDA medium was supplemented with different methyl anthranilate concentrations $\quad\left(0, \quad 100 \mu \mathrm{mol} \cdot \mathrm{L}^{-1}, \quad 500 \mu \mathrm{mol} \cdot \mathrm{L}^{-1}, \quad 1\right.$ $\mathrm{mmol} \cdot \mathrm{L}^{-1}, 10 \mathrm{mmol} \cdot \mathrm{L}^{-1}$ ) of methyl anthranilate. $5 \mu \mathrm{L}$ conidial suspension $\left(1 \times 10^{6}\right.$ conidia. $\left.\mathrm{mL}^{-1}\right)$ of $V$. dahliae and hypha discs $(5 \mathrm{~mm})$ of $B$. cinerea were inoculated on the center of PDA medium containing methyl anthranilate. The fungi were then cultured in an incubator at $25^{\circ} \mathrm{C}$. After 1 week, colony diameters were measured.

\section{Accession numbers}

Sequence data from this article can be found in the CottonGen database (http://www.cottongen.org) or GenBank databases under the following accession numbers: GbTRP1, Gbar_D12G029450; GhUB7, Gh_A11G0969; GhPR1, Gh_A12G0274; GhPR2, Gh_D06G2277; GhPR3, Gh_D01G1683; GhPR4, Gh_D13G1816; GhPR5, Gh_ D12G2247; GhPR10, Gh_D04G1399.

\section{Supplementary information}

Supplementary information accompanies this paper at https://doi.org/10. 1186/s42397-019-0034-1

Additional file 1: Table. S1. Primers used in this study. The bold and underlined parts are adaptor sequence; the primers for RT-qPCR are marked by 'RL'. Figure S1. Alignment of the amino acid sequences of GbTRP1 (Gbar_D12G029450) with AtTRP1 (AT5G17990). Yellow box indicates chloroplast transit peptide (CTP). Figure S2. Detection of SA contents in the leaves of TRV:00 and TRV:TRP1 plants. A: High-performance liquid chromatography (HPLC) profiles of SA extracted from leaf samples of TRV:00 and TRV:TRP1 seedlings. The number superimposed on each peak is the retention time (RT), and three extra peaks (RT $=5.35,6.74$, 7.11) are indicated in the TRV:TRP1 samples. B: Detection of SA contents in TRV:00 and TRV:TRP1 plants 14 days after infiltration. The values represent the means \pm SD from three biological replicates $\left({ }^{* *} p<0.01\right.$, Student's $t$-test).

\section{Acknowledgments}

We would like to thank Prof. THOMMA Bart (Laboratory of Phytopathology, Wageningen University and Research Center, The Netherlands) for providing VIGS vectors and Prof. JIAN Guiliang (Institute of Plant Protection, Chinese Academy of Agricultural Sciences, China) for providing $V$. dahliae strain V991'. Language revision by Prof. LINDSEY Keith (Department of Biosciences, Durham University, Durham, UK) is appreciated.

\section{Authors' contributions}

Zhang XL and Zhu LF designed the experiment. Miao YH performed the research, analyzed the data and drafted the manuscript. Zhang $X L$ revised the manuscript. All authors read and approved the final manuscript.

\section{Funding}

Supports by China Agricultural Research System (CARS-15-04) are appreciated.

\section{Availability of data and materials}

Not applicable.

Ethics approval and consent to participate

Not applicable

\section{Consent for publication}

Not applicable.

\section{Competing interests}

The authors declare that they have no competing interests.

\section{Author details}

${ }^{1}$ National Key Laboratory of Crop Genetic Improvement, Huazhong Agricultural University, Wuhan 430070, Hubei, China. ${ }^{2}$ Hubei University of Chinese Medicine, Wuhan 430065, Hubei, China.

Received: 14 May 2019 Accepted: 23 September 2019

Published online: 12 November 2019

\section{References}

Ahuja I, Kissen R, Bones AM. Phytoalexins in defense against pathogens. Trends Plant Sci. 2012;17(2):73-90.

Bednarek P, Piślewska-Bednarek M, Svatoš A, et al. A glucosinolate metabolism pathway in living plant cells mediates broad-spectrum antifungal defense. Science. 2009;323(5910):101. https://doi.org/10.1126/science.1163732.

Cai Y, He XH, Mo J, et al. Molecular research and genetic engineering of resistance to Verticillium wilt in cotton. Afr J Biotechnol. 2009;8(25):7363-72.

Carpenter CW. The Verticillium wilt problem. Phytopathology. 1914;4(6):393.

Chae HS, Kieber JJ. Eto Brute. Role of ACS turnover in regulating ethylene biosynthesis. Trends Plant Sci. 2005;10(6):291-6.

Chai Q, Shang X, Wu S, et al. 5-aminolevulinic acid dehydratase gene dosage affects programmed cell death and immunity. Plant Physiol. 2017;175(1):51128.

Chen Z, Agnew JL, Cohen JD, et al. Pseudomonas syringae type III effector AvrRpt2 alters Arabidopsis thaliana auxin physiology. Proc Natl Acad Sci U S A. 2007;104(50):20131-6.

Crawford IP. Evolution of a biosynthetic pathway: the tryptophan paradigm. Annu Rev Microbiol. 1989;43(1):567-600.

Fradin EF, Haliem A, Masini L, et al. Interfamily transfer of tomato Ve1 mediates Verticillium resistance in Arabidopsis. Plant Physiol. 2011;156(4):2255-65.

Gao W, Long L, Zhu LF, et al. Proteomic and virus-induced gene silencing (VIGS) analyses reveal that gossypol, brassinosteroids, and jasmonic acid contribute to the resistance of cotton to Verticillium dahliae. Mol Cell Proteomics. 2013; 12(12):3690-703.

Gao X, Wheeler T, Li Z, et al. Silencing GhNDR1 and GhMKK2 compromises cotton resistance to Verticillium wilt. Plant J. 2011;66(2):293-305.

Guo WF, Jin L, Miao YH, et al. An ethylene response-related factor, GbERF1-like, from Gossypium barbadense improves resistance to Verticillium dahliae via activating lignin synthesis. Plant Mol Biol. 2016;91(3):305-18.

Hartmann M, Zeier T, Bernsdorff F, et al. Flavin monooxygenase-generated NHydroxypipecolic acid is a critical element of plant systemic immunity. Cell. 2018;173(2):456-69.

He X, Zhu LF, Wassan GM, et al. GhJAZ2 attenuates cotton resistance to biotic stresses via inhibiting the transcriptional activity of GhbHLH171. Mol Plant Pathol. 2017;19(4):896-908.

Hu Q, Min L, Yang X, et al. Laccase GhLac1 modulates broad-spectrum biotic stress tolerance via manipulating phenylpropanoid pathway and jasmonic acid synthesis. Plant Physiol. 2018a;176(2):1808-23.

Hu Q, Zhu LF, Zhang XN, et al. GhCPK33 negatively regulates defense against Verticillium dahliae by phosphorylating GhOPR3. Plant Physiol. 2018b;178(2): 876 
Iven T, Konig S, Singh S, et al. Transcriptional activation and production of tryptophan-derived secondary metabolites in arabidopsis roots contributes to the defense against the fungal vascular pathogen Verticillium longisporum. Mol Plant. 2012:5(6):1389-402.

Jin $S$, Zhang $X$, Nie $Y$, et al. Identification of a novel elite genotype for in vitro culture and genetic transformation of cotton. Biol Plant. 2006;50(4):519-24.

Kawchuk LM, Hachey J, Lynch DR, et al. Tomato Ve disease resistance genes encode cell surface-like receptors. Proc Natl Acad Sci U S A. 2001;98(11): $6511-5$.

Last RL, Fink GR. Tryptophan-requiring mutants of the plant Arabidopsis thaliana. Science. 1988;240(4850):305-10.

Li C, He X, Luo XY, et al. Cotton WRKY1 mediates the plant defense-todevelopment transition during infection of cotton by Verticillium dahliae by activating JASMONATE ZIM-DOMAIN1 expression. Plant Physiol. 2014;166(4): 2179-94.

Li YB, Han LB, Wang HY, et al. The thioredoxin GbNRX1 plays a crucial role in homeostasis of apoplastic reactive oxygen species in response to Verticillium dahliae infection in cotton. Plant Physiol. 2016;170(4):2392-406.

Liu L, Zhang W, Zhou Y, et al. Resistance of cotton and tomato to Verticillium dahliae from cotton is independent on Ve1. Scientia Sinica Vitae. 2014b;44(8): 803 .

Liu T, Song T, Zhang X, et al. Unconventionally secreted effectors of two filamentous pathogens target plant salicylate biosynthesis. Nat Commun. 2014a;5:4686.

Lu HP, Luo T, Fu HW, et al. Resistance of rice to insect pests mediated by suppression of serotonin biosynthesis. Nat Plants. 2018. https://doi.org/10. 1038/s41477-018-0152-7

Mace ME, Stipanovic RD, Bell AA. Toxicity and role of terpenoid phytoalexins in Verticillium wilt resistance in cotton. Physiol Plant Pathol. 1985;26(2):209-18.

Miao YH, Xu L, He X, et al. Suppression of tryptophan synthase activates cotton immunity by triggering cell death via promoting SA synthesis. Plant J. 2019. https://doi.org/10.1111/tpj.14222 .

Møldrup ME, Geu-Flores F, Halkier BA. Assigning gene function in biosynthetic pathways: Camalexin and beyond. Plant Cell. 2013;25(2):360-7.

Radwanski ER, Last RL. Tryptophan biosynthesis and metabolism: biochemical and molecular genetics. Plant Cell. 1995;7(7):921-34.

Rose AB, Casselman AL, Last RL. A phosphoribosylanthranilate transferase gene is defective in blue fluorescent Arabidopsis thaliana tryptophan mutants. Plant Physiol. 1992;100(2):582-92.

Shan L, He P. Pipped at the post: Pipecolic acid derivative identified as SAR regulator. Cell. 2018;173(2):286-7.

Song Y, Liu L, Wang Y, et al. Transfer of tomato immune receptor Ve1 confers Ave1-dependent Verticillium resistance in tobacco and cotton. Plant Biotechnol J. 2018;16(2):638-48.

Sun LQ, Zhu LF, Xu L, et al. Cotton cytochrome P450 CYP82D regulates systemic cell death by modulating the octadecanoid pathway. Nat Commun. 2014;5: 5372.

Van Loon LC, Van Strien EA. The families of pathogenesis-related proteins, their activities, and comparative analysis of PR-1 type proteins. Physiol Mol Plant Pathol. 1999:55(2):85-97.

Xu L, Zhang W, He X, et al. Functional characterization of cotton genes responsive to Verticillium dahliae through bioinformatics and reverse genetics strategies. J Exp Bot. 2014;65(22):6679-92.

$\mathrm{Xu} \mathrm{L}$, Zhu LF, Tu LL, et al. Lignin metabolism has a central role in the resistance of cotton to the wilt fungus Verticillium dahliae as revealed by RNA-Seqdependent transcriptional analysis and histochemistry. J Exp Bot. 2011;62(15): $5607-21$.

Zeier J. New insights into the regulation of plant immunity by amino acid metabolic pathways. Plant Cell Environ. 2013;36(12):2085-103.

Zhu LF, Tu LL, Zeng FC, et al. An improved simple protocol for isolation of high quality RNA from Gossypium spp. suitable for cDNA library construction. Acta Agron Sin. 2005;31:1657-9.

Ready to submit your research? Choose BMC and benefit from:

- fast, convenient online submission

- thorough peer review by experienced researchers in your field

- rapid publication on acceptance

- support for research data, including large and complex data types

- gold Open Access which fosters wider collaboration and increased citations

- maximum visibility for your research: over $100 \mathrm{M}$ website views per year

At $\mathrm{BMC}$, research is always in progress.

Learn more biomedcentral.com/submissions 